\title{
Genetic Toggle Switch Without Cooperative Binding
}

\author{
Azi Lipshtat ${ }^{1}$, Adiel Loinger ${ }^{2}$, Nathalie Q. Balaban ${ }^{2}$ and Ofer Biham ${ }^{2}$ \\ 1 Department of Pharmacology and Biological Chemistry, Mount Sinai \\ School of Medicine, New York, NY 10029, USA \\ ${ }^{2}$ Racah Institute of Physics, The Hebrew University, Jerusalem 91904, Israel
}

\begin{abstract}
Genetic switch systems with mutual repression of two transcription factors are studied using deterministic and stochastic methods. Numerous studies have concluded that cooperative binding is a necessary condition for the emergence of bistability in these systems. Here we show that for a range of biologically relevant conditions, a suitable combination of network structure and stochastic effects gives rise to bistability even without cooperative binding.
\end{abstract}

PACS numbers: PACS: 87.10.+e, 87.16.-b 
Recent advances in quantitative measurements of gene expression at the single-cell level 1, 2] have brought new insight on the importance of stochastic fluctuations in genetic circuits 3]. Populations of genetically identical cells show variability due to fluctuations. The role of fluctuations is enhanced due to the discrete nature of the transcription factors and their binding sites, which may appear in low copy numbers 4, 5]. Stochastic behavior may invoke oscillations [6, 7] and spatio-temporal patterns [8, 9, 10], which are unaccounted for by macroscopic chemical rate equations. Genetic circuits with feedback mechanisms often exhibit bistability, namely, two distinct stable states which can be switched either spontaneously or by an external signal [11, 12, 13]. To qualify as a switch, the spontaneous switching rate must be much lower than the rates of the relevant processes in the cell, namely transcription, translation, binding and unbinding of transcription factors. In particular, genetic switches such as the phage $\lambda$ switch, enable cells to adopt different fates [14]. The toggle switch is a simple genetic circuit that consists of two proteins, $A$ and $B$, with concentrations $[A]$ and $[B]$, respectively, which negatively regulate each other's synthesis (by concentration we mean the average copy number of proteins per cell). The production of protein $A$ is negatively regulated by protein $B$, through binding of $n$ copies of $B$ to the $A$ promoter (and vice versa). This process can be modeled by a Hill function, which reduces the production rate of $A$ by a factor of $1+k[B]^{n}$, where $k$ is a parameter and $n$ is the Hill coefficient [15]. In case that $n=1$ the binding of a single protein is sufficient in order to perform the negative regulation, while for $n>1$ the cooperative binding of two or more proteins is required. In numerous studies of the toggle switch system it was concluded that cooperative binding is a necessary condition for the emergence of the two distinct stable states characteristic of a switch [16, 17, 18, 19]. It was also observed that in presence of cooperative binding, stochastic effects contribute to the broadening of the parameter range in which bistability appears [20].

In this letter we show that stochastic effects enable bistability even without cooperative binding of the transcription factors to the operator, namely for Hill coefficient $n=1$. Furthermore, bistability takes place even when the active proteins appear in high copy numbers. These results emphasize the necessity of stochastic methods in the analysis of genetic networks, even under conditions of high concentrations.

The mutual repression circuit, referred to as the general switch [18], is described by the 
rate equations

$$
\begin{aligned}
{[\dot{A}] } & =g_{A}\left(1-\left[r_{B}\right]\right)-d_{A}[A]-\alpha_{0}[A]\left(1-\left[r_{A}\right]\right)+\alpha_{1}\left[r_{A}\right] \\
{[\dot{B}] } & =g_{B}\left(1-\left[r_{A}\right]\right)-d_{B}[B]-\alpha_{0}[B]\left(1-\left[r_{B}\right]\right)+\alpha_{1}\left[r_{B}\right] \\
{\left[\dot{r_{A}}\right] } & =\alpha_{0}[A]\left(1-\left[r_{A}\right]\right)-\alpha_{1}\left[r_{A}\right] \\
{\left[\dot{r_{B}}\right] } & =\alpha_{0}[B]\left(1-\left[r_{B}\right]\right)-\alpha_{1}\left[r_{B}\right]
\end{aligned}
$$

where $g_{X}\left(\mathrm{~s}^{-1}\right), X=A, B$, is the maximal production rate of protein $X$ and $d_{X}\left(\mathrm{~s}^{-1}\right)$ is its degradation rate. For simplicity, we ignore the mRNA level and take the processes of transcription and translation as a single step of synthesis [21]. The bound repressors are considered as separate species $r_{X}$ and their concentrations are given by $\left[r_{X}\right]$, providing much insight into the repression process [22]. Here, $r_{A}$ is a bound $A$ protein that monitors the production of $B$, while $r_{B}$ is a bound $B$ protein that monitors the production of $A$. Since there is a single promoter of each type, $0 \leq\left[r_{X}\right] \leq 1$. The parameter $\alpha_{0}\left(\mathrm{~s}^{-1}\right)$ is the binding rate of proteins to the promoter and $\alpha_{1}\left(\mathrm{~s}^{-1}\right)$ is the dissociation rate.

It is commonly assumed that the binding-unbinding processes are much faster than other processes in the circuit, namely $\alpha_{0}, \alpha_{1} \gg d_{X}, g_{X}$. This means that the relaxation times of $\left[r_{X}\right]$ are much shorter than other relaxation times in the circuit. Under this assumption, one can take the time derivatives of $\left[r_{X}\right]$ to zero, even if the system is away from steady state. This brings the rate equations to the standard Michaelis-Menten form

$$
\begin{aligned}
& {[\dot{A}]=g_{A} /(1+k[B])-d_{A}[A]} \\
& {[\dot{B}]=g_{B} /(1+k[A])-d_{B}[B],}
\end{aligned}
$$

where $k=\alpha_{0} / \alpha_{1}$ is the repression strength. For a given population of free $X$ repressors, the parameter $k$ controls the value of $\left[r_{X}\right]$. The limit of weak repression, $\left[r_{X}\right] \ll 1$, is obtained when $k[X] \ll 1$, while the limit of strong repression, $\left[r_{X}\right] \simeq 1$, is obtained for $k[X] \gg 1$. These equations turn out to have one positive steady-state solution, thus at the level of rate equations this system does not exhibit bistability. For symmetric parameters, where $g_{A}=g_{B}=g$ and $d_{A}=d_{B}=d$, this solution is $[A]=[B]=\left[(1+4 k g / d)^{1 / 2}-1\right] / 2 k$.

In order to account for stochastic effects, the master equation approach [3, 20, 23] is applied. In the master equation, the dynamic variables are the probabilities $P\left(N_{A}, N_{B}, r_{A}, r_{B}\right)$ for a cell to include $N_{X}$ copies of free protein $X$ and $r_{X}$ copies of the bound $X$ repressor, 
where $N_{X}=0,1,2, \ldots$, and $r_{X}=0,1$. The master equation for the mutual repression circuit takes the form

$$
\begin{aligned}
& \dot{P}\left(N_{A}, N_{B}, r_{A}, r_{B}\right)=g_{A} \delta_{r_{B}, 0} P\left(N_{A}-1, N_{B}, r_{A}, r_{B}\right)+g_{B} \delta_{r_{A}, 0} P\left(N_{A}, N_{B}-1, r_{A}, r_{B}\right) \\
& +d_{A}\left(N_{A}+1\right) P\left(N_{A}+1, N_{B}, r_{A}, r_{B}\right)+d_{B}\left(N_{B}+1\right) P\left(N_{A}, N_{B}+1, r_{A}, r_{B}\right) \\
& -\left(g_{A} \delta_{r_{B}, 0}+g_{B} \delta_{r_{A}, 0}\right) P\left(N_{A}, N_{B}, r_{A}, r_{B}\right)-\left(d_{A} N_{A}+d_{B} N_{B}\right) P\left(N_{A}, N_{B}, r_{A}, r_{B}\right) \\
& +\alpha_{0}\left[\left(N_{A}+1\right) \delta_{r_{A}, 1} P\left(N_{A}+1, N_{B}, 0, r_{B}\right)+\left(N_{B}+1\right) \delta_{r_{B}, 1} P\left(N_{A}, N_{B}+1, r_{A}, 0\right)\right] \\
& +\alpha_{1}\left[\delta_{r_{A}, 0} P\left(N_{A}-1, N_{B}, 1, r_{B}\right)+\delta_{r_{B}, 0} P\left(N_{A}, N_{B}-1, r_{A}, 1\right)\right] \\
& -\alpha_{0}\left(N_{A} \delta_{r_{A}, 0}+N_{B} \delta_{r_{B}, 0}\right) P\left(N_{A}, N_{B}, r_{A}, r_{B}\right)-\alpha_{1}\left(\delta_{r_{A}, 1}+\delta_{r_{B}, 1}\right) P\left(N_{A}, N_{B}, r_{A}, r_{B}\right),
\end{aligned}
$$

where $\delta_{i, j}=1$ for $i=j$ and 0 otherwise. The $g_{X}$ terms account for the production of proteins. The $d_{X}$ terms account for the degradation of free proteins, while the $\alpha_{0}\left(\alpha_{1}\right)$ terms describe the binding (unbinding) of proteins to (from) the promoter site. The average copy numbers $\langle X\rangle$, where $X=N_{A}, N_{B}, r_{A}, r_{B}$, are given by $\langle X\rangle=\sum X P\left(N_{A}, N_{B}, r_{A}, r_{B}\right)$ where the sum is over all integer values of $N_{A}$ and $N_{B}$ up to a suitable cutoffs and over $r_{A}, r_{B}=0,1$. Note that for distributions that are skewed or exhibit several peaks, the average does not reflect the actual behavior in a single cell.

To analyze the role of fluctuations in this circuit we have calculated the probability distribution $P\left(N_{A}, N_{B}\right)=\sum_{r_{A}, r_{B}} P\left(N_{A}, N_{B}, r_{A}, r_{B}\right)$. We used the symmetric parameters $g=0.05\left(\mathrm{~s}^{-1}\right)$, which correspond to average production time of 20 seconds, and $d=0.005$ $\left(\mathrm{s}^{-1}\right)$ which means degradation time of 200 seconds, in agreement with experimental results 24]. To examine a broad range of relevant values of $k$ we performed two sets of simulations. In the first set we chose $\alpha_{1}=0.5\left(\mathrm{~s}^{-1}\right)$ and varied $\alpha_{0}$, while in the second set we chose $\alpha_{0}=0.5\left(\mathrm{~s}^{-1}\right)$ and varied $\alpha_{1}$. We confirmed that the population of free proteins depends only on the ratio, $k$.

Under conditions in which the promoter sites are empty most of the time, namely $r_{X} \ll 1$, the repression is weak and the steady state solution exhibits coexistence of $A$ and $B$ proteins in the cell. In this case the distribution $P\left(N_{A}, N_{B}\right)$ exhibits a single peak [Fig. 1(a)]. In this case, the values of $\left\langle N_{A}\right\rangle$ and $\left\langle N_{B}\right\rangle$ obtained from the master equation coincide with [A] and $[B]$, obtained from the rate equations. For strong repression, the distribution $P\left(N_{A}, N_{B}\right)$ exhibits a peak in which the $A$ population is suppressed and a peak in which the $B$ population is suppressed, as expected for a bistable system. However, a third peak appears near the 
origin, in which both populations of free proteins diminish [Fig. 1(b)]. This peak represents a dead-lock situation, caused by the fact that both $A$ and $B$ repressors can be bound simultaneously, each bringing to a halt the production of the other specie. This result is in contrast to the rate equations which exhibit a single solution, $[A]=[B]$, for the entire range of parameters. Below, we present three biologically sensible variants of the circuit in which the third peak is suppressed, giving rise to a bistable switch.

Consider the exclusive switch, where there is an overlap between the promoters of $A$ and $B$ and thus no room for both to be occupied simultaneously. Such a situation is encountered in nature, for example, in the lysis-lysogeny switch of phage $\lambda$ [14]. It was shown that in presence of cooperative binding, the exclusive switch is more stable than the general switch [18]. This is because in the exclusive switch the access of the minority specie to the promoter site is blocked by the dominant specie. Here we show that in the exclusive switch, stochastic effects give rise to bistability even without cooperativity between the transcription factors. To model this system recall that $\left[r_{A}\right]\left(\left[r_{B}\right]\right)$ can be defined as the fraction of time in which the promoter is occupied by a bound $A(B)$ protein. The fraction of time in which the promoter is vacant is $1-\left[r_{A}\right]-\left[r_{B}\right]$. Incorporating this into Eq. (II) gives rise to the following modification: in the $\alpha_{0}$ terms, each appearance of $\left[r_{A}\right]$ or $\left[r_{B}\right]$ should be replaced by $\left[r_{A}\right]+\left[r_{B}\right]$. For symmetric parameters, the resulting equations still exhibit a single solution, in which $[A]=[B]$ and $\left[r_{A}\right]=\left[r_{B}\right]$. The Michaelis-Menten equations for the exclusive switch are given by Eqs. (2), where in the first equation $k$ is replaced by $k /(1+k[A])$ and in the second equation it is replaced by $k /(1+k[B])$. To account for the discreteness of the transcription factors and their fluctuations, the master equation should be applied, with the constraint that $P\left(N_{A}, N_{B}, 1,1\right)=0$. It takes the form of Eq. (3), except that in the $\alpha_{0}$ and $\alpha_{1}$ terms, each time $\delta_{r_{A}, j}\left(\delta_{r_{B}, j}\right)$ appears it should be multiplied by $\delta_{r_{B}, 0},\left(\delta_{r_{A}, 0}\right)$. In the exclusive switch, under conditions of weak repression, $P\left(N_{A}, N_{B}\right)$ exhibits a single peak [Fig. 2(a)], for which $\left\langle N_{A}\right\rangle$ and $\left\langle N_{B}\right\rangle$ coincide with $[A]$ and $[B]$, respectively. For strong repression, the distribution $P\left(N_{A}, N_{B}\right)$ exhibits two peaks. In one peak the $A$ population is suppressed, while in the other peak the $B$ population is suppressed, as expected for a bistable system [Fig. 2(b)]. The dead-lock situation is impossible in this system.

To examine the time dependence of the populations of free proteins in a single cell, we have performed Monte Carlo simulations, based on the master equation for the exclusive switch. In Fig. 3 we present the copy numbers of free and bound $A$ and $B$ proteins vs. time. 
The population size of the dominant specie is in the range of 20-60, while the minority specie is almost completely suppressed. The typical switching time is around $10^{5}$ seconds.

Consider a different variant of the genetic switch, which exhibits bound-repressor degradation (BRD). Even a low degradation rate, $d_{r}$, of the bound repressors tends to remove the mutual suppression of both species, and gives rise to a binary switch. The rate equations that describe this circuit are identical to Eq. (10), except that a degradation term of the form $-d_{r}\left[r_{A}\right]\left(-d_{r}\left[r_{B}\right]\right)$ is added to the equation for $\left[\dot{r}_{A}\right]\left(\left[\dot{r}_{B}\right]\right)$. For symmetric parameters, the Michaelis-Menten form of these equations, applicable in the limit of fast switching, is given by Eq. (2) where $k=\alpha_{0} /\left(\alpha_{1}+d_{r}\right)$ and $d$ is replaced by an effective degradation rate $d_{\mathrm{eff}}=d+d_{r} k /(1+k[A])$ in the first equation and by the analogous term in the second equation. This equation exhibits a bifurcation at $k_{c}=\left(d / d_{r}\right)\left(\sqrt{g}+\sqrt{d_{r}}\right) /\left(\sqrt{g}-\sqrt{d_{r}}\right)$, in which the symmetric solution $[A]=[B]$ becomes unstable, giving rise to two stable solutions in which one specie is dominant and the other is suppressed (Fig. 圆, inset). We thus find that in case that bound repressors exhibit degradation, bistability appears even at the level of rate equations. The emergence of bistability can be attributed to the fact that the effective degradation rate for the minority specie is larger than for the dominant specie, enhancing the difference between the population sizes. The master equation for this circuit is obtained by adding the term $d_{r}\left[\left(\delta_{r_{A}, 0}-\delta_{r_{A}, 1}\right) P\left(N_{A}, N_{B}, 1, r_{B}\right)+\left(\delta_{r_{B}, 0}-\delta_{r_{B}, 1}\right) P\left(N_{A}, N_{B}, r_{A}, 1\right)\right]$ to Eq. (3). This term represents transitions of the cell from $r_{X}=1(X=A, B)$ to $r_{X}=0$, without changing the number of free proteins. The degradation of bound repressors gives rise to suppression of the peak near the origin, leading to the emergence of bistability.

A third variant of the genetic switch exhibits protein-protein interactions (PPI) such that an $\mathrm{A}$ protein and a $\mathrm{B}$ protein may form an $\mathrm{AB}$ complex, which is not active as a transcription factor. This circuit exhibits bistability within a range of parameters, both for the rate equations and for the master equation.

We have calculated the switching time using the master equation, for an initial state that includes only free A proteins. The distribution $P\left(N_{A}, N_{B}\right)$ vs. time was calculated and the function $f(t)=P\left(N_{A}>N_{B}\right)-P\left(N_{A}<N_{B}\right)$ was found to decay exponentially according to $f(t)=\exp (-t / \tau)$, where $\tau$ is defined as the switching time. In Fig. $⿴$ we present the switching time $\tau$, obtained from the master equation vs. $k$ for the exclusive switch (o) and for the BRD switch $(\times)$. We also examined the dependence of $\tau$ on the copy number, $N$, of the dominant specie. For the exclusive switch, we found that when $d$ is varied, $\tau \sim N^{2}$, while 
in case that $g$ is varied, $\tau \sim N$. This dependence is weaker than found for the cooperative switch [18].

The results presented in this paper (except for Fig. [3) were obtained by direct integration of the master equation rather than by Monte Carlo methods [25]. Direct integration is much more efficient and provides more accurate results, without the need to accumulate statistics. Recent improvements in the methodology enable to use direct integration for complex networks that involve large numbers of active proteins [26], which will enable to go beyond elementary circuits into simulations of complete networks.

In contrast to previous knowledge that bistability requires cooperative binding of transcription factors, we have shown that bistability is possible without cooperative binding. We have analyzed three variants of the genetic toggle switch, that exhibit bistability without cooperative binding. The first circuit is the exclusive switch, in which the two promoter sites cannot be occupied simultaneously. The second circuit exhibits degradation of bound repressors, while in the third circuit free $A$ and $B$ proteins may form a complex which is not active as a transcription factor. Rate equations predict a single stable state in the first circuit and bistability in the second and third circuits. However, the master equation predicts bistability in all the three circuits. These findings are not limited to cases in which proteins exist in low numbers, but are due to the low copy number of the promoter itself. The results presented here are expected to have significant implications on the understanding of non-genetic variability in cell populations, and may shed new light on the way cells differentiate despite uniform environmental conditions.

N.Q.B. was supported by the Center of Complexity of the Horowitz Foundation and the Bikura Program of the Israel Science Foundation.

[1] M.B. Elowitz, A.J. Levine, E.D. Siggia and P.S. Swain, Science 297, 1183 (2002).

[2] E.M. Ozbudak, M. Thattai, I. Kurtser, A.D. Grossman and A. van Oudenaarden, Nature Genetics 31, 69 (2002).

[3] H.H. McAdams and A. Arkin, Proc. Natl. Acad. Sci. US 94, 814 (1997); A. Arkin, J. Ross and H.H. McAdams, Genetics 149, 1633 (1998).

[4] A. Becskei and L. Serrano, Nature 405, 590 (2000). 
[5] M. Kaern, T.C. Elston, W.J. Blake and J.J. Collins, Nature Reviews Genetics 6, 451 (2005).

[6] J.M. Vilar, H.Y. Kueh, N. Barkai and S. Leibler, Proc. Natl. Acad. Sci. US 99, 5988 (2002).

[7] T. Zhou, L. Chen and K. Aihara, Phys. Rev. Lett. 95, 178103 (2005).

[8] N.M. Shnerb, Y. Louzoun, E. Bettelheim and S. Solomon, Proc. Natl. Acad. Sci. US 97, 10322 (2000).

[9] M. Howard and A.D. Rutenberg, Phys. Rev. Lett. 90, 128102 (2003).

[10] Y. Togashi and K. Kaneko, Phys. Rev. E 70, 020901 (2004).

[11] M.R. Atkinson, M.A. Savageau, J.T. Myers and A.J. Ninfa, Cell 113, 597 (2003).

[12] E.M. Ozbudak, M. Thattai, H.N. Lim, B.I. Shraiman, A. van Oudenaarden, Nature 427, 737 (2004).

[13] P. Francois and V. Hakim, Phys. Rev. E 72, 031908 (2005).

[14] M. Ptashne, A Genetic Switch: Phage $\lambda$ and Higher Organisms, 2nd edition. (Cell Press and Blackwell Scientific Publications, Cambridge, MA, 1992).

[15] Hill-function models are simplifications of rate-law equations. When derived directly from rate laws, $n$ is expected to take only integer values. However, when these models are used for fitting empirical data, $n$ is a fitting parameter which may take non-integer values.

[16] T.S. Gardner, C.R. Cantor and J.J. Collins, Nature 403, 339 (2000).

[17] J.L. Cherry and F.R. Adler, J. Theor. Biol. 203, 117 (2000).

[18] P.B. Warren and P.R. ten Wolde, Phys. Rev. Lett. 92, 128101 (2004); J. Phys. Chem. B 109, $6812(2005)$.

[19] A.M. Walczak, M. Sasai and P. Wlynes, Biophysical J. 88, 828 (2005).

[20] T.B. Kepler and T.C. Elston, Biophysical Journal 81, 3116 (2001).

[21] Extended circuits that include the mRNA level have also been studied. It was found that there is a perfect agreement between the rate equation results for the simplified and the extended circuits, and excellent agreement with only slight difference in peak shapes in the master equation.

[22] A. Lipshtat, H.B. Perets, N. Q. Balaban and O. Biham, Gene 347, 265 (2005).

[23] J. Paulsson and M. Ehrenberg, Phys. Rev. Lett. 84, 5447 (2000); J. Paulsson, Nature 427, 415 (2004).

[24] M.B. Elowitz and S. Leibler, Nature 403, 335 (2000).

[25] D.T. Gillespie, J. Chem. Phys. 81, 2340 (1977). 
[26] A. Lipshtat and O. Biham, Phys. Rev. Lett. 93, 170601 (2004). 
FIG. 1: The probabilities $P\left(N_{A}, N_{B}\right)$ for the general switch, under conditions of (a) weak repression $(k=0.005)$ where there is one symmetric peak and (b) strong repression $(k=50)$ where three peaks appear, one dominated by $A$, the second dominated by $B$ and the third in which both species are mutually suppressed. The weights of the three peaks are about the same.

FIG. 2: The probabilities $P\left(N_{A}, N_{B}\right)$ for the exclusive switch, under conditions of (a) weak repression $(k=0.005)$ where there is one symmetric peak and (b) strong repression $(k=50)$ where bistability is observed.

FIG. 3: The populations of free and bound $A$ and $B$ proteins vs. time, obtained from Monte Carlo simulations of the exclusive switch with the parameters $g=0.2, d=0.005, \alpha_{0}=0.2$ and $\alpha_{1}=0.01$. The bistable behavior is clearly observed, where the population size of the dominant specie is between 20-60 and the other specie is nearly diminished. Failed switching attempts are clearly seen.

FIG. 4: The switching time vs. the repression strength, $k$, for the exclusive switch (o) and for the case in which bound repressors exhibit degradation $(\times)$. For the bistable range (roughly $k>1$ ) the switching time increases as $k$ is increased. The inset shows the steady state solution for $[A]$ and $[B]$ vs. $k$, obtained from the rate equations for the BRD switch. Note that for the BRD switch, the parameter $\alpha_{0}$ varies, while $d_{r}=d$ and $\alpha_{1}=0.01$ are held fixed. 

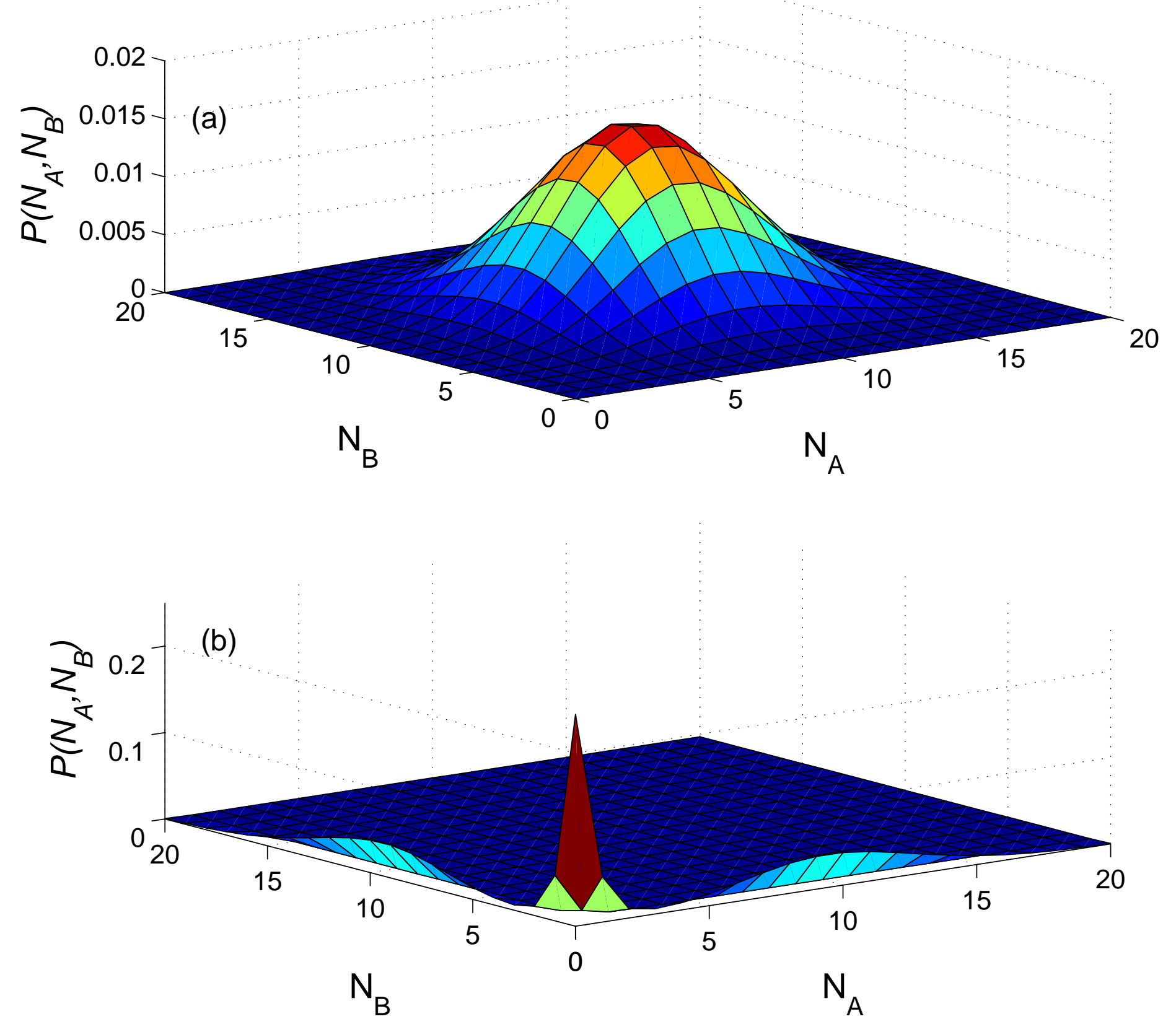

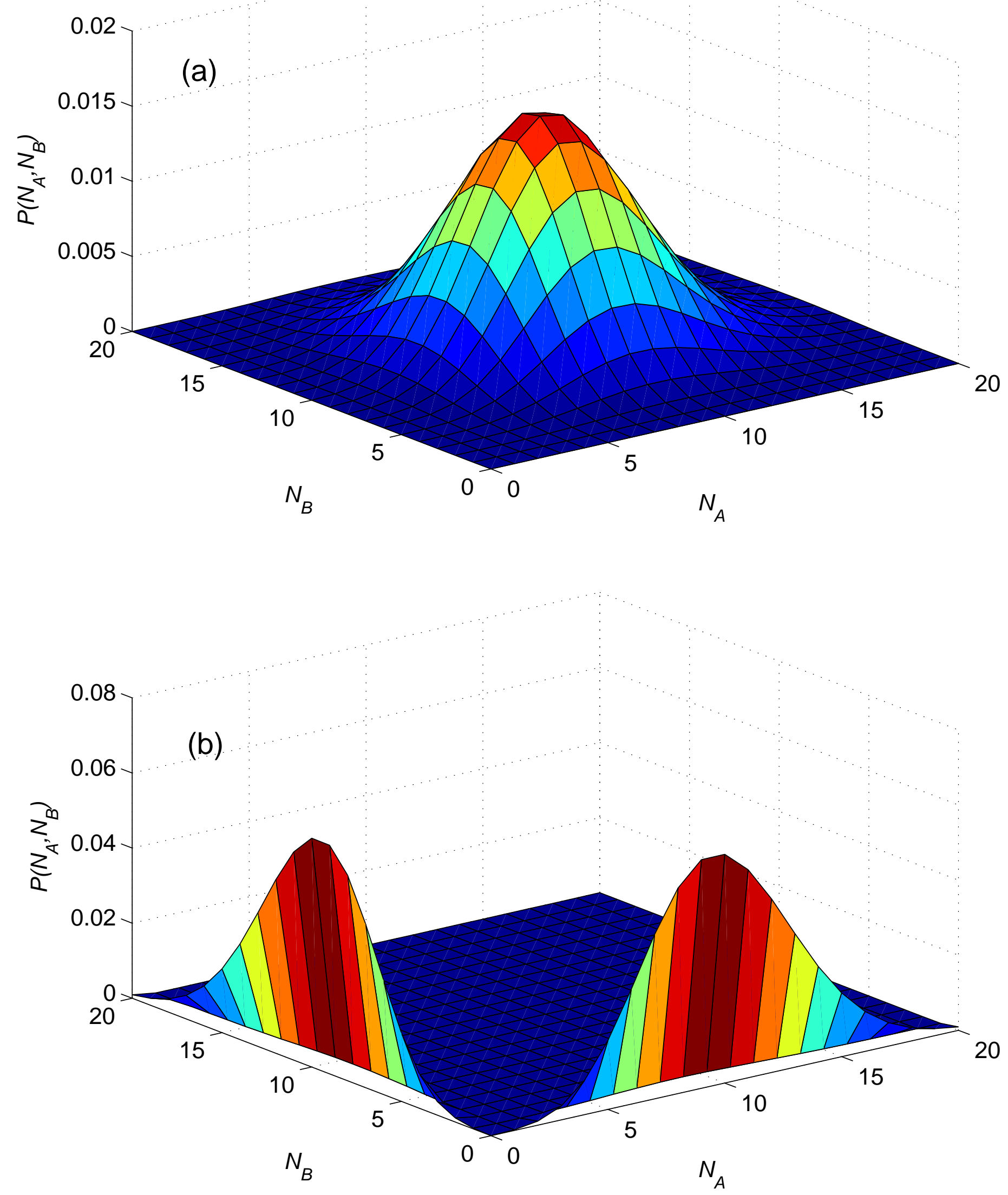

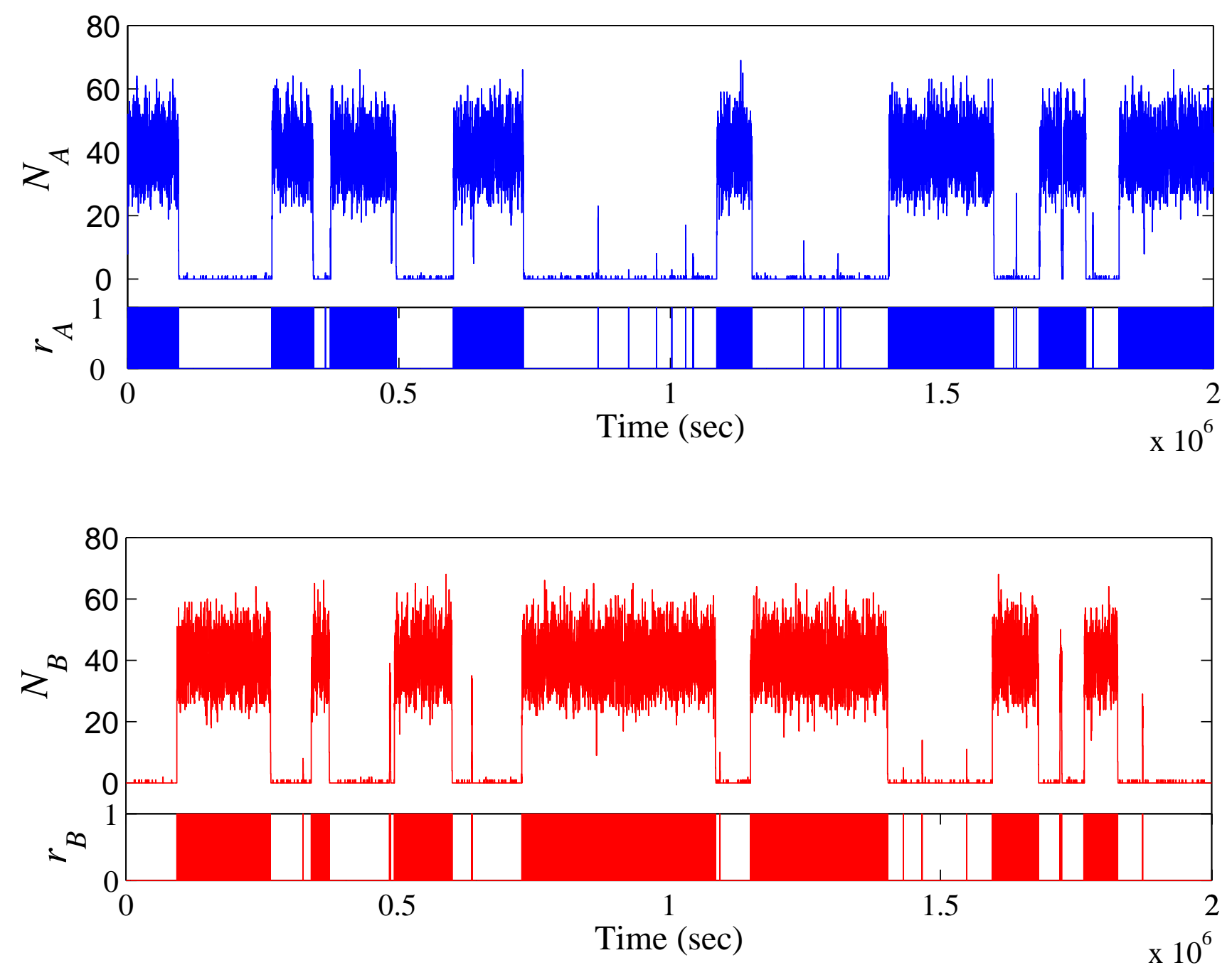